\title{
Design of 3D Shooting Game
}

\author{
Ping Wang \\ College of Information Engineering, Jiangxi University of Technology, Jiangxi Nanchang
}

Keywords: Engine; Cutting; DirectX.

\begin{abstract}
In recent years, due to the breakthrough in 3D hardware graphics technology, the real-time depicted pictures have been increasingly sophisticated, and 3D games have been more diversified and closer to the real world. In this paper, the author used primarily Visual C ++6.0 as a development tool to understand and master the knowledge about Windows programming technique, DirectX programming technique and physical modeling. The author develops a DirectX graphics engine and produces a 16-bit game on this basis. In the engine, the author uses double buffering and page switching technologies to maintain a smooth running of the game at a 30FPS refresh rate; prepares the cutting function to draw the game on the screen from the internal storage in one piece rather than points one by one, so the game runs faster. At the end of the game, the author also prepares the resource release program to rationally use the system resources.
\end{abstract}

\section{Introduction}

Game Development History. Electronic game was originated in the West. The international game industry has a history of 42 years, while the game publishing industry in China mainland began in 1994. In the 1980s, the game industry in China Taiwan region was booming and some companies of game development and publishing with full growth of channel appeared. In the mid-1990s, EA's "Command \& Conquer" in America and "The Legend of Sword and Fairy" in Taiwan, China entered the mainland market, stirring up a whirlwind of computer games. In the late 1990s, China's game development capability gradually improved. Many studios were springing up, and some local companies joined one after another. There were many excellent works, such as Qiandao Company's "Red Cliff", Jinshan's "JX Online" and Aoshi's "Metal Knight". Unfortunately, due to immature market, limited operating level, especially the impact of piracy, the majority of research and development companies were difficult to survive, making adjustments. The development of domestic games entered into a low ebb. In order to support the national game industry, strengthen international exchanges and cooperation, protect intellectual property and lead the healthy consumption, the General Administration of Press and Publications and China Council for Promotion of International Trade proposed to hold the China International Digital Interactive Entertainment Products and Technical Applications Exhibit (hereinafter referred to as China-Joy). In order to improve the status of game publishing industry, regulate gaming publication market, eliminate the negative factors in industrial development, strengthen ties between enterprises and the government, safeguard the legitimate rights and interests of Chinese game publishing enterprises, and promote exchanges and cooperation between Chinese and foreign game industries and make Chinese game publishing industry more healthy 
and prosperous, the Publishers Association of China proposed to set up the game working committee in a timely manner, and began to prepare for establishment at the end of 2002.

This space shooting game is a simple shooting game. The space enemies are 3D solid. In this game, players can use the mouse to control the shooting cross. Click the left mouse button to shoot, and press the ESC to exit. The game rule is very simple: players must use the particle beam weapon to eliminate enemies; if the number of enemies that escape reaches 25 , the game will end. If the game is restarted, players only need to press the key of Enter.

\section{Demand Analysis}

Feasibility Analysis. Economic Analysis.

With the rapid development of computer technology, a variety of PC games are emerging. To meet their needs, players are willing to spend certain money on entertainment. Why do players play PC games rather than visit museum or watch movies? What is the specialty of PC game relative to people's pursuit of other amusements? What do PC games provide that other activities cannot provide? According to general understanding, the attractive point of a game is enabling us to strengthen our own specialties and making our artistic forms different from other forms, which cannot be met by other media. To achieve success, it's required to use these specialties, sublimate them and create the best game. The reasons are as follows:

Players need challenges

Players need communications

Players need independent experience

Players need showy right

Players need emotional experience

Players need fantasy

Technical Feasibility.

Visual $\mathrm{C}++$ is the 4GL software development tool launched by American Microsoft Company and has become one of the senior program design languages most widely applied in China.

DirectX is an application programming interface (API), which allows games or multimedia applications based on windows platform to achieve higher efficiency, strengthens $3 \mathrm{~d}$ graphic and sound effects, and provides a common hardware driven standard for designers. Meanwhile, game developers do not need to write different drive programs for each brand. The complexity of installation and setup of hardware is reduced. Maybe it is quite unclear in this way. Actually, in the literal sense, Direct is direct; X means many. Thus, the purpose of DirectX is to provide direct services for a lot of software.

Operational Feasibility.

With the development of science and technology, more and more people rely on computer, and the computer is more and more popular. Similarly, network becomes more and more practical. Many people will seek information, buy items, hold video conference and do entertainment on the Internet.

Analysis of Game players. The most popular games among game players

Players who play chess and card as well as role play games account for about $60 \%$; players who play the leisure action and puzzle interesting games respectively account for $50 \%$ each; the percentage of players who always play role play games is the highest, up to $33.5 \%$; players 
who always play chess and card, leisure action and puzzle interesting games respectively account for $20.7 \%, 13.5 \%$ and $11.2 \%$.

Purpose of game players.

Like players of role play games, the players of leisure action games mainly play for pure entertainment, accounting for $56 \%$, followed by players for exercising intelligence, accounting for $17.2 \%$. The entertainment of games is the important factor that attracts players.

It can be seen from analysis of factors emphasized by players of leisure action games that the enjoyment of games occupies the highest percentage (48.3\%). In addition, players pay attention to game picture and sound effect as well as operating speed.

\section{System Design and Realization}

Overall Game Design. 1. Initialization.Memory allocation, resources access and data loading from disk, etc.

2. Enter into game cycle.Users will continue to carry out actions in this part unit exiting the cycle

3. Read player input.This part deals with player input, or stores it in the buffer for AI and game logic

4. Execute AI and game logic.This part contains the theme of game code, which will execute artificial intelligence, physical system and general game logic, and draw the next frame on the screen according to results.

5. Render next frame.In this part, according to player input as well as the results of execution of game AI and logic, the next game animation frame will be generated. This kind of picture is generally drawn in the off-screen buffer. Therefore, users cannot see the rendering process. The next frame will be quickly copied to the visible zone to produce animation effect.

6. Cycle.This part is relatively simple. Players only need to return to the beginning of the game cycle and execute the whole cycle

7. Close.This is the end of the game, meaning that users have exited the game theme or game cycle and returned to the operating system. However, similar to other software, all occupied sources need releasing before returning to the operating system and the system need clearing. This phase can only be controlled by the keyboard, but later by the mouse, so that the joy of game can be improved.

The following is the status switching diagram of game cycle logic: 


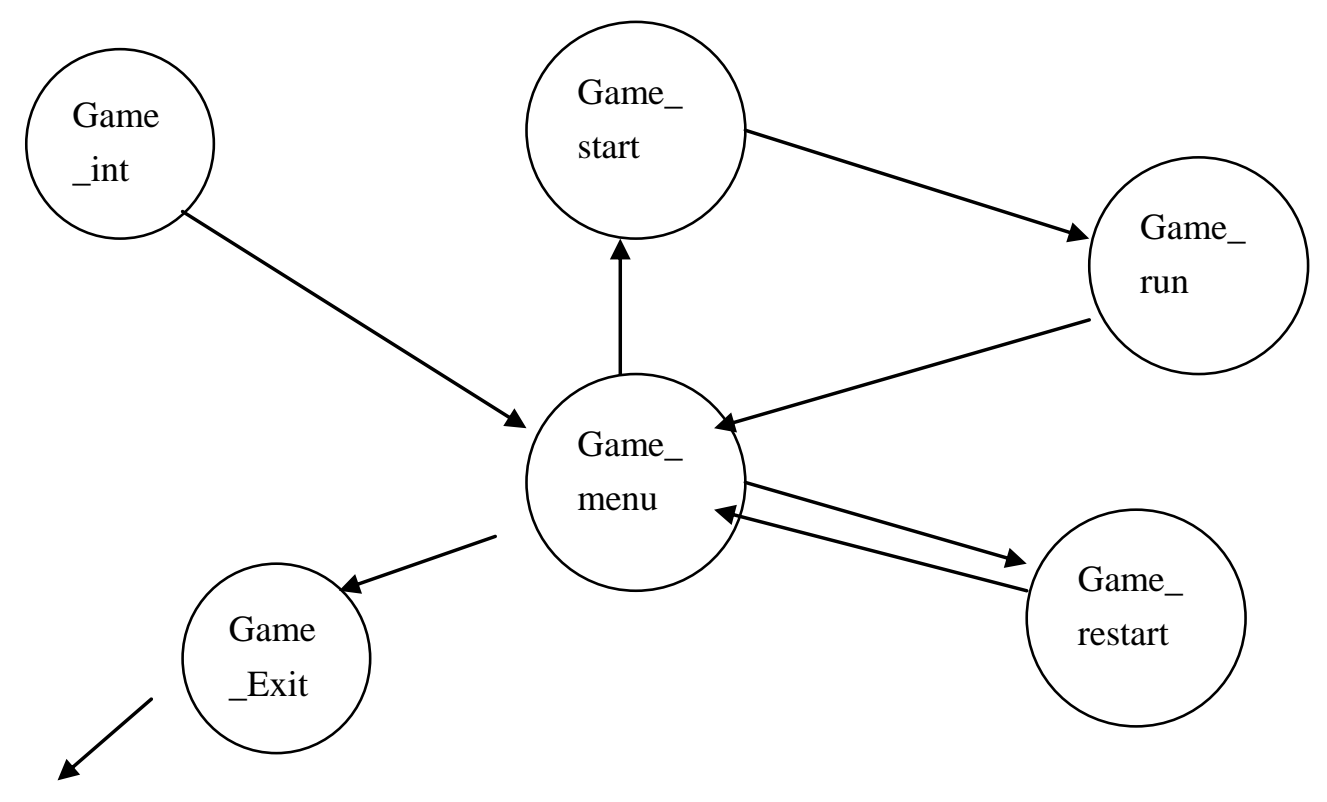

Fig. 1 Status Switching Diagram of Game Cycle Logic

Establish a Windows Program. The objective of this design is to write the 3D game operating in the windows platform. Therefore, much programming knowledge about windows is not involved. In actual, only a framework-windows program is needed. It opens the window, deals with messages and transfers main game cycle. That's all.

Establish Windows Class. Windows is a real object-oriented operating system, so many concepts and process in windows are from $\mathrm{c}$ and $\mathrm{c}++$. Windows class is one of them. In windows, window, control and listbox are essentially windows, and their difference lies in the definition of their classes. Each windows class is a description of window type that can be handled by the windows.

Register Windows Class. After defining windows class and storing it in the winclass, it is necessary to enable windows operating system to know this class through registration. The registration is completed by RegisterClass (\&winclass) and only the pointer pointing to new class is accepted as the parameter. After the registration of class, it can be used for creating windows.

Event Processor. Event processor is a callback function. When an event needs processing in the window, windows operating system uses this function in the main event cycle.

Establish and Use DriectX COM Interface. It is known that the COM object is the collection of some interfaces. These interfaces are some function pointers, namely VTABLE. Therefore, for DirectX COM object, it needs to be established to obtain an interface pointer, and then this interface shall be invoked with appropriate grammar. Firstly, DirectDraw needs to be used and three works need to be completed.

It is necessary to load and register COM object and DLL in the operating process of DirectDraw. This work is completed by Directx installation program.

It is necessary to contain DDRAWLIB import library in Win 32 program in order to connect the used package function to the program. 
It is necessary to contain DDRAWH document in the program, so that the compiler can see the head information of DirectDraw as well as function prototype and data type.

Tailoring. Tailoring is the most important theme in 3D graphics. If the geometric structure is not correctly tailored, it cannot be correctly displayed and then many problems will be caused, such as abnormality of zero removal and memory breakdown. To this end, tailoring is necessary.

Object space tailoring refers to tailoring of geometric stricter consisting of basic pattern primitives in the specific tailoring zone. The tailoring zone can be 2D or 3D. The important thing is that object space tailoring is conducted in the mathematic space according to the mathematic expressions of polygon and pattern primitives. The advantage of object space tailoring is that its theory is very clear: tailor object or polygon list according to $2 \mathrm{D}$ or $3 \mathrm{D}$, and then transmit the tailored polygon to the next process of 3D assembly line.

The problem of object space tailoring lies in detail. In the engine, triangle is always processed. The tailoring must be based on $2 \mathrm{D}$ rectangle or $3 \mathrm{D}$ visual object. It can produce polygon with over 3 vertices. In other words, the tailoring may add new vertices to the triangle, thus making it into square or other polygons. Therefore, it is necessary to segment the triangle again. This handling process may be unclear. If the engine is designed to support any square, this problem will not occur. However, the optimization has been made basically only based on supporting triangle. Secondly, in the object space system, the tailoring involves calculation of intersections between straight lines as well as intersections between straight line and plane.

Game AI Design.

Purpose of Game AI Design.

1. Add challenges to players

In many old games, there are not so many AI signs. For example, in the game "Super Mario", the types of monsters as well as order and walking modes are not changed according to the change of player behavior. If we spend some time on this game to know the appearance points of these monsters, we can easily pass the game (of course, to avoid this situation the game designer designs sophisticated map to make up for deficiency of AI). In many games, enemy NPC moves not according to unchanged route. NPC makes appropriate judgment and takes action according to player behavior. In the larger range, NPC has certain "independence", for example, NPC in CS.

For players, to deal with complex AI enemy, they need higher skills. The challenge is added to some extent.

2. Create a more realistic virtual world

Maybe many people have played the original CS. In fact, NPC in the original CS is relatively stupid, because they can only walk with the fixed route and their ability to respond to emergencies is relatively poor. In general, as long as we attack some NPC, the NPC hardly has opportunities to survive even if our shooting skills are poor. They never turn around. After a series of corrections, NPC in CS cannot be easily handled. After attacked, they will quickly turn around and attack, or even "speak", send messages to other NPC for support. This action is more similar to player behavior.

In some previous games, NPC could be blocked in front of a stone or tree, walking against the tree, but could not take a detour. Thus, players will look down on this game. "This game is too false" or "this game is boring", they may sigh. As a result, this game will be uninstalled. In 
today's games, this situation rarely occurs. The calculation of multiple "way-finding methods" has been the most fundamental requirement for game programmers.

3. Add playability

The reason why people prefer to play CS, Warcraft and other games on game platform-battle net is that fighting with people can give the game more styles and larger unpredictability. Players seek for the result of unpredictability. If we know the end of a movie at the beginning, we will lose our enthusiasm for it. The game is the same case.

Enabling NPC to have AI and simulate people's thinking can give players larger unpredictability or give a surprise to them. In fact, some AI routines are given to NPC for selection. For instance, according to HP, select AI algorithms such as "brave to go", "fighting with withdrawal" and "escape first". Thus, players cannot accurately speculate the action of NPC. The playability is developed.

4. Support other functions

For example, if we play a game and control a role to enter a city, we find that enemies in this city are different from AI enemies in other places. Even if HP is few, they will continue to attack our role. Players will have a doubt, namely what drives them to be so crazy? It is easy to stimulate our curiosity in another way. Then this game will tell the players some information, for instance, the village was attacked by foreign people, so people hated invasion and were brave to sacrifice. In this way, it is easy for players to accept. AI design indirectly describes the story.

The explosion effect in this game can be called a kind of AI. If players hit the spaceship but the spaceship does not respond, then the joy of this game will be largely reduced. However, the explosion is not as exciting as the author expected. The algorithm is that: copy the polygon grid of invasive spaceship to the explosion grid that can be easily handled. The aim is to facilitate moving or changing the polygons of the object in the explosion phase. In the explosion phase, throw out the polygons (random explosion) at different angles and rates.

Design of main components of game.

\section{Starry sky}

To achieve starry sky, some tricks are used. Since the engine does not support stars, they must be rendered manually and the sight distance and window size same in the engine shall be used in the perspective equation. To a large extent, the logic here is very simple. The stars similar to particles are very tiny. Players will not notice whether the lighting calculation or $\mathrm{z}$ ranking is executed for them. However, it is indeed necessary to add the support for particle system in the engine.

\section{Energy weapon}

This is a kind of cool special effect. At first, straight lines were used to represent weapons. But then the idea was changed to make particle (plasma) energy weapons look like lightning, which would certainly realize good effect. To achieve this, I wrote such a function: it received two terminals (draw energy bind between them). The energy bind is cut into several sections and the maximum amplitude is used as parameter. Amazingly, the expected effect was obtained after only one attempt.

3. Collision detection and target tracking

Collision detection is very easy. Basically, there is a shooting cross in the $2 \mathrm{D}$ space. Players move it on the screen, but the object is in the $3 \mathrm{D}$ space. Therefore, it is necessary to project the 
surrounding frame of each spaceship in the 2D screen space, and then detect whether the center of shooting cross is in the surrounding frame. To this end, when the author calculated the surrounding frame of each spaceship, 4 vertices of the surrounding frame were projected and whether the center of shooting cross was in the surrounding frame was detected.

4. Explosion

The explosion effect is not as exciting as expected. The algorithm is that: copy the polygon grid of invasive spaceship to the explosion grid that can be easily handled. However, the author did not use the vertex combination, but copied polygons one by one. Therefore, some vertices were repeated. The aim was to facilitate moving or changing the polygons of the object in the explosion phase. In the explosion phase, throw out the polygons (random explosion) at different angles and rates.

\section{Font engine}

The speed of GDI is very slow. It is feasible to use it to indicate status or other debugging information. However, its speed is too slow for indicating game text. Therefore, a simple font engine was written for this game and two functions were used. They lied in the main function rather in the library block. The first function is as follows:

int Load_Bitmap_Font(char *fontfile, BOB_PTR font);

It loads a bitmap that contains template font. The font must contain 64 characters, which are divided into 4 lines, 16 characters per line. Each character must be $16^{*} 16$ pixels. The first character is a space character. In addition, when using the above font loading function, it is necessary to transmit an empty BOB to store characters. Each character occupies an animation cell.

The second function is used in any location of the screen to indicate a character string. When using this function, it is necessary to transmit the BOB that contains font bitmap, coordinates $\mathrm{x}$ and $\mathrm{y}$, and to indicate the character string, horizontal and vertical distances as well as a renderable surface.

6. Lighting in game

There are 4 light sources in the engine. :

One environmental light source

One white sun. It is a point light source, located in the top right corner of the game space.

One red fixed star. It is a point light source, located in the left bottom of the game space. The surrounding fixed stars are represented by the bitmaps.

One energy releaser. It is a point light source, located in several hundred units in front of the spaceship controlled by the player. Only when the player uses weapons, this light source will be activated.

\section{Conclusions}

This design is 3D shooting game design. The development of this game is based on the deep understanding of knowledge about picture processing, through comprehensive investigation and analysis and by full use of theoretical knowledge and skills of computer major. After a series of demand analysis, system design, system implementation, system testing and other application system development processes, this design has basically completed the task of graduation project. In function, the space shooting game developed basically reach the expected result as well as entertainment effects. This shooting game in this design is highly 
specialized and has higher requirements for $\mathrm{C}++$ programming and digital image processing technology and algorithm.

\section{Acknowledgements}

This work was financially supported by project of Technology Department of Jiangxi Province [No 20143BBM26048] and the project of Technology Department of Jiangxi Province [No 2013BBE50051] also gives us lots of help.

\section{References}

[1] Arerbuch A , Lazar D , Israeli M . "Image Compression Using Wavelet Transform and Multiresonlution Decomposition. " IEEE Trans , Image Processing , 1996.5 (1):4 15

[2] Yang Shuying. VC++ Image Processing Program Design. Beijing: Tsinghua University Press, 2003: 11-12

[3] Xi Ching, Zhang Chunlin. Visual C++ 6.0 Use Programming Technique. Beijing: China Water Power Press, 1999: 11-12

[4] Zhang Shujin. Image Processing and Analysis. Beijing: Tsinghua University Press, 1999: 1813182

[5] Zhang Honglin. Digital Image Pattern Recognition Technology. Beijing: People's Posts and Telecommunications Press, 2003: 1123159

[6] Herbert Schildt. C ++ Shortcut Course. Beijing: Tsinghua University Press, 2004: 43-64

[7] Yang Mingjun. C++ Practical Training Course. Beijing: People's Posts and Telecommunications Press, 2002: 4-15

[8] Eugenc Kain. MFC Classic Q\&A. Beijing: China Electric Power Press, 2001: 45-78 